\title{
Desenvolvimento de uma aplicação mobile para apoio na aprendizagem de tarefas do cotidiano de crianças com Transtorno do Espectro Autista
}

\author{
Cristiane Alberto da Silva Conrad ${ }^{1}$, Jean Felipe Patikowski Cheiran ${ }^{1}$ (orientador) \\ ${ }^{1}$ Curso de Engenharia de Software - Campus Alegrete \\ Universidade Federal do Pampa (UNIPAMPA) \\ Avenida Tiaraju, 810 - 97.546-550 - Alegrete - RS - Brazil \\ cristiane.conrad@gmail.com, jeancheiran@unipampa.edu.br
}

\begin{abstract}
This work shows the creation of a game to support children with Autism Spectrum Disorder (ASD) in developing their ability to perform everyday tasks. Accessibility guidelines, accessibility recommendations and interviews with experts assisted the game design. Techniques adopted in this work include paper prototyping, user testing, interviews and MEEGA+ questionnaire. The game was developed in Unity platform and Personal Scrum process drove the development. Tests involved five children with ASD, three adults, and two experts. The contributions include the indication of adopted accessibility guidelines, the thought of faced challenges, and the game as an available product.
\end{abstract}

Resumo. Esse trabalho mostra a criação de um jogo para auxiliar crianças com Transtorno do Espectro Autista (TEA) no desenvolvimento de habilidades para atividades do cotidiano. Diretrizes e recomendações de acessibilidade $e$ entrevistas com especialistas embasaram o game design. Técnicas usadas no trabalho incluem protótipos em papel, testes com usuários, entrevistas, e questionário MEEGA+. O jogo foi desenvolvido na plataforma Unity e o processo Personal Scrum guiou o desenvolvimento. Os testes envolveram cinco crianças com TEA, três adultos responsáveis e duas especialistas. As contribuições incluem a indicação das diretrizes de acessibilidade adotadas, a reflexão sobre desafios enfrentados, e o jogo como produto disponível.

\section{Introdução}

Segundo pesquisas, o número de pessoas com deficiência vem crescendo no Brasil [IBGE 2010]. Apesar do número de pessoas com Transtornos de Espectro Autista (TEA) não se destacar como outros, este grupo não deve ser deixado de lado. As pessoas com estes transtornos podem apresentar problemas de socialização e dificuldades relacionadas à realização de tarefas do cotidiano por terem um ritmo mais lento [Cardoso 2014].

Quando pesquisado acerca da prevalência do TEA no Brasil, não foi encontrada uma pesquisa que buscasse abordar números focando no país como um todo. Já com relação à região sul do país, entre os meses de dezembro de 2016 e maio de 2017 , constatou-se que a estimativa de prevalência para cada 10.000 crianças no Rio Grande do Sul era de 3,31 [Beck 2017]. Já em Santa Catarina o número subiu para 3,94 em cada 10.000 crianças e, no Paraná, 4,32. 
VIII Congresso Brasileiro de Informática na Educação (CBIE 2019)

Anais dos Workshops do VIII Congresso Brasileiro de Informática na Educação (WCBIE 2019)

O TEA se caracteriza por uma condição de desenvolvimento neurológico, ou seja, desordens complexas no desenvolvimento do cérebro. Esses distúrbios são especialmente caracterizados pela dificuldade na comunicação social e comportamentos repetitivos [Moreira et al. 2016]. Durante diferentes momentos da vida, pessoas com TEA podem ter dificuldade de aprendizagem por conta da dificuldade de interação social. Este problema pode se manifestar tanto em tarefas escolares quanto no aprendizado de atividades da vida diária como, por exemplo, escovar os dentes ou tomar banho.

A tecnologia pode ser um fator de ajuda no desenvolvimento de crianças com TEA. Nesse sentido, muitos aplicativos e jogos têm sido desenvolvidos pensando no aprendizado dessas crianças. Ainda, [Hussain et al. 2016] afirmam que as crianças com TEA tendem a ser mais receptivas a utilizar jogos em dispositivos móveis devido à interface de toque na tela e portabilidade do dispositivo. Esses recursos fornecem uma interação mais natural e direta, auxiliando a criança a manter seu foco. Alguns exemplos de jogos para sistemas Android são: Funny Food, Nico Explore Your Bathroom, TEO - Autismo, e AieLLo. Em geral, esses jogos são desenvolvidos com focos específicos, como cuidados de higiene pessoal, melhoria de atenção e memória, e estimulação de comunicação. Não foi encontrado um jogo que aborde o cotidiano como um todo.

Assim, o objetivo deste trabalho é o desenvolvimento de um jogo mobile para ampliar a autonomia de crianças com TEA na realização de tarefas diárias, baseando-se em recomendações de acessibilidade para pessoas com TEA e na perspectiva de especialistas. A Figura 1 apresenta uma versão simplificada das etapas realizadas neste trabalho.

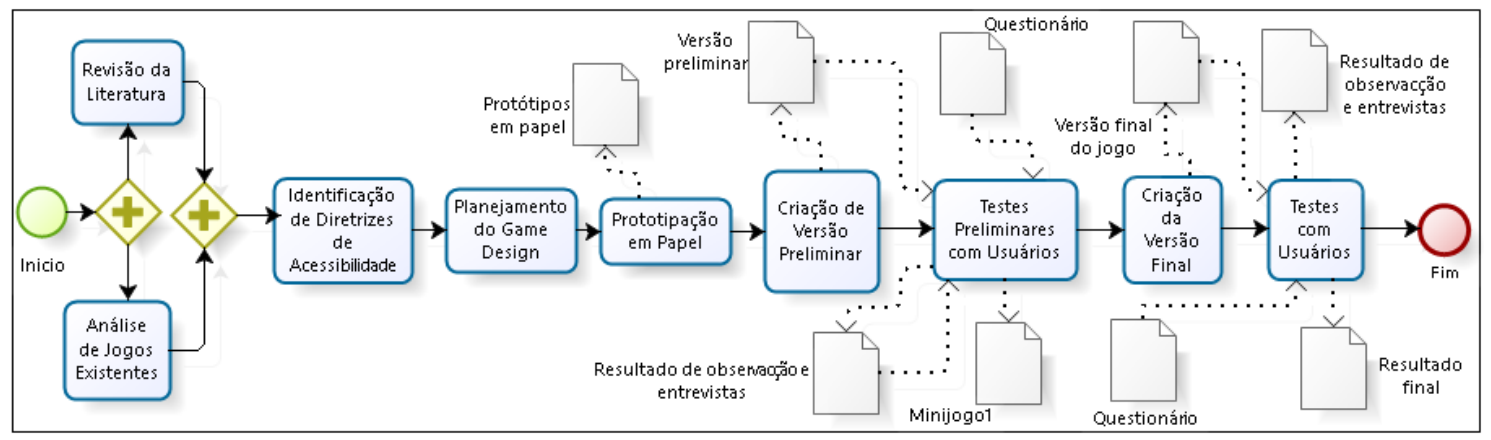

Figura 1. Desenho simplificado da pesquisa

\section{Fundamentação}

\subsection{Desenvolvimento de Jogos Digitais}

Em um primeiro momento, para que se possa definir desenvolvimento de jogos digitais e suas etapas, é necessário definir o que é um jogo. Os jogos são instrumentos que viabilizam o ato de jogar, e nos quais as regras ou os objetivos ou ambos impõem algum nível de estrutura para as interações [Rabin 2011]. Assim, o jogo é um sistema que interage com os jogadores, envolvendo-os em um conflito artificial mediado por um conjunto de regras e tendo um objetivo determinado [Salen and Zimmerman 2012].

Não apenas o objetivo mediado pelas regras dentro do jogo é algo importante para caracterizá-lo, mas também o objetivo de se jogar aquele jogo como entreter-se, socializar, aprender, etc. Indiferente ao tipo de jogo, reconhece-se que os jogos servem como 
VIII Congresso Brasileiro de Informática na Educação (CBIE 2019)

Anais dos Workshops do VIII Congresso Brasileiro de Informática na Educação (WCBIE 2019)

ferramenta para a apreensão, desenvolvimento e manutenção de habilidades específicas desde a coordenação visomotora até os conhecimentos históricos de um período -, atuando como uma ferramenta de apoio para o aprendizado do indivíduo.

Segundo [Abrantes 2010], os jogos educativos favorecem a construção do conhecimento e das habilidades, mas devem ser bem planejados e aplicados com objetivos definidos, levando em conta a idade e as limitações de cada aluno. Entre as atividades que podem ser propostas em jogos educativos para crianças, podemos citar: reconhecimento de cores, formas, números, letras e objetos de uso diário.

O processo de desenvolvimento de jogos é semelhante ao desenvolvimento de software, porém inclui características vistas em outras mídias e incomuns nos softwares tradicionais. Novas atividades (que envolvem produção de arte visual, composição musical, modelagem $2 \mathrm{D}$ ou $3 \mathrm{D}$, escrita de narrativas, balanceamento de desafios e outras) incorporadas em processos de desenvolvimento de software tradicionais [Sommerville 2011] fundamentam processos de desenvolvimento de jogos digitais [Rabin 2011], incluindo adaptações que atendem equipes altamente multidisciplinares.

\subsection{Transtorno do Espectro Autista}

No que diz respeito ao enquadramento do Transtorno do Espectro Autista (TEA) como deficiência, [Faders and do Rio Grande do Sul 2011] define que, em virtude de suas características, o TEA se enquadra no grupo das deficiências psicossociais. A deficiência psicossocial consta na classificação da Organização Mundial da Saúde (OMS) e a abrangência do termo psicossocial está definida na Classificação Internacional de Funcionalidade Deficiência e Saúde (CIF), sob a identificação b122: "São as funções mentais de modo geral, que, à medida que se desenvolvem ao longo da vida, são necessárias para o entendimento e integração construtiva da função mental que leva à formação de habilidades sociais para se estabelecer as interações sociais de reciprocidade em termos de significado e propósito" [Organization 2017].

Conforme [Association et al. 2014], a gravidade do TEA é dividida em níveis e se baseia em seus prejuízos relacionados à habilidade de comunicação social e em padrões de comportamento restritos e repetitivos, classificando-se o grau do transtorno conforme a necessidade de suporte e/ou a dependência. [Association et al. 2014] ainda define que os níveis considerados variam entre nível 3 (severo), nível 2 (moderado) e nível 1 (leve), podendo variar ao longo do tempo e de um tratamento.

Durante a realização deste trabalho, foram encontrados grupos de diretrizes em três áreas para a acessibilidade em jogos que podem ser aplicadas a pessoas com TEA. Nestas áreas, podemos destacar as Diretrizes de Acessibilidade Cognitiva conforme propostas por [AbleGamers 2018] e [GAG 2018].

$\mathrm{Na}$ área de Design de Interação (IxD) e propostas para pessoas com TEA, podemos destacar o trabalho de [Hussain et al. 2016], que leva em conta as três dimensões do Design de Interação, divididas em Forma, Conteúdo e Comportamento.

Por fim, na área de Acessibilidade Web, pode-se destacar o Guia de Acessibilidade de Interfaces Web com foco em aspectos do autismo (GAIA) que é um conjunto de recomendações elaborado para auxiliar no desenvolvimento de websites com foco no uso de dispositivos multitoque proposto por [Britto 2016]. 
VIII Congresso Brasileiro de Informática na Educação (CBIE 2019)

Anais dos Workshops do VIII Congresso Brasileiro de Informática na Educação (WCBIE 2019)

\section{Trabalhos relacionados}

A busca destes trabalhos se deu a partir da formulação de uma string de busca (em português e em inglês) utilizando palavras-chave ligadas ao tema deste trabalho: jogos $\mathbf{E}$ crianças E (autismo OU “síndrome de Down”) E (web OU “dispositivos móveis")

As bases utilizadas, por sua disponibilidade de acesso e relevância, foram Scopus ${ }^{2}$, ACM Digital Library ${ }^{3}$ e artigos publicados no Simpósio Brasileiro de Jogos e Entretenimento Digital (SBGames) $)_{4}^{\sqrt{3}}$, sendo que, neste último, a busca foi realizada análise manual dos últimos 5 anos nos anais do SBGames. Após a busca e a aplicação de critérios para filtrar os trabalhos com alta relevância e afinidade, destacaram-se os trabalhos abaixo.

Jecripe 2: estimulação da memória, atenção e sensibilização fonológica em crianças com Síndrome de Down - O jogo proposto por [Brandão and Joselli 2015] tem por objetivo auxiliar na estimulação de crianças com necessidades especiais, tendo potencial para ser utilizado no tratamento de pacientes por profissionais da área de saúde.

ComFiM: A Cooperative Serious Game to Encourage the Development of Communicative Skills between Children with Autism - A proposta de [Ribeiro et al. 2014] foi a de desenvolver um jogo sério para tablets com o intuito de desenvolver e melhorar as habilidades de comunicação de crianças com autismo.

Using Serious Games to Improve Therapeutic Goals in Children with Special Needs - A proposta do trabalho de [Durango et al. 2015] é verificar se existe diferença entre a realização de atividades por modo tradicional e por uso de jogos digitais para a realização de atividades educacionais similares.

Interaction Design Principles for Edutainment Systems: Enhancing the Communication Skills of Children with Autism Spectrum Disorders - A pesquisa de [Hussain et al. 2016] aborda a análise da interface de cinco aplicações de dispositivos móveis, apontando quinze sugestões de melhorias com respeito ao design de interfaces para atender às necessidades específicas dos usuários alvo de forma mais eficiente.

Gyroscope-Based Game Revealing Progress of Children with Autism - Segundo [Kolakowska et al. 2017], este trabalho propõe uma forma de automação da medição de progressos de crianças com TEA utilizando métricas de comportamento derivadas de monitoramento de giroscópio e estado de jogo e de aprendizado de máquina.

Além da pesquisa de trabalhos acadêmicos, também foi feita uma pesquisa na web e na loja de aplicativos Google Play Store por jogos educativos e jogos para crianças com autismo. A comparação destes jogos é apresentada na Tabela 1.

Embora existam outros esforços de desenvolvimento de jogos para crianças com TEA, os trabalhos científicos encontrados não se concentram nas atividades cotidianas das crianças e os produtos disponíveis na Google Play Store não indicam sistematização científica detalhada associada ao seu desenvolvimento.

\footnotetext{
${ }^{1} \mathrm{O}$ termo síndrome de Down fazia parte da pesquisa, mas foi posteriormente excluído.

${ }^{2}$ Disponível em https: / / www. scopus. com/.

${ }^{3}$ Disponível em https://dl.acm.org.

${ }^{4}$ Disponível em https: / / www.sbgames.org

${ }^{5}$ Foram feitas buscas separadas, utilizando strings de busca "jogos educativos" e "jogos autismo", para que se pudesse ter uma ideia dos tipos de jogos desenvolvidos para cada caso.
} 
VIII Congresso Brasileiro de Informática na Educação (CBIE 2019)

Anais dos Workshops do VIII Congresso Brasileiro de Informática na Educação (WCBIE 2019)

Tabela 1. Tabela de comparação de jogos.

\begin{tabular}{llll}
\hline Jogo & Foco & Idade & Gratuito \\
\hline Funny Food & $\begin{array}{l}\text { Competências básicas de lógica, atenção, } \\
\text { memória, cores e formas. }\end{array}$ & Parte \\
\hline $\begin{array}{l}\text { Nico Explore } \\
\text { Your Bathroom }\end{array}$ & Tarefas relacionadas à higiene. & $2-6$ & Sim \\
\hline Pepi Bath Lite & Tarefas relacionadas à higiene. & 4 ou mais & Parte \\
\hline $\begin{array}{l}\text { Jogos Educativos } \\
\text { Crianças 5 }\end{array}$ & $\begin{array}{l}\text { Alfabeto; memória; formas; cores e ta- } \\
\text { manhos; pintura e quebra-cabeças. }\end{array}$ & Sim \\
\hline Pictogramas.es & Comunicação. & n.d. & Sim \\
\hline TEO - Autismo & $\begin{array}{l}\text { Comunicação, socialização e comporta- } \\
\text { mento. }\end{array}$ & n.d. & Sim \\
\hline Autastico & $\begin{array}{l}\text { Formas, expressões faciais, números, co- } \\
\text { res e resolver quebra-cabeças. }\end{array}$ & Não \\
\hline AieLLo & Vocabulário. & Livre & Não \\
\hline
\end{tabular}

\section{Materiais e Métodos}

Essa pesquisa se caracteriza como qualitativa e exploratória [Sampieri et al. 2013].

O universo de participantes foi composto por três crianças com TEA atendidas em um consultório de fonoaudiologia particular, duas crianças com TEA alunas da Associação de Pais e Amigos dos Excepcionais (APAE) de Alegrete/RS, uma fonoaudióloga, uma terapeuta ocupacional, e três adultos responsáveis pelas crianças.

Para o desenvolvimento do jogo, foram empregadas as técnicas de Personal Scrum [Costa 2016], histórias de usuário e prototipação em papel. Como ferramentas adicionais, foram utilizadas o Trello para acompanhamento das histórias de usuário, a plataforma Unity para o desenvolvimento do jogo e as ferramentas Adobe Photoshop e Premiere Pro para criação de gráficos e vídeos.

Entrevistas com questões abertas foram usadas para levantamento de requisitos e recomendações de acessibilidade com uma especialista - terapeuta ocupacional. Após a construção dos jogos, foram realizados testes com usuários [Barbosa and Silva 2010] utilizando um protocolo de teste e a observação das atividades. Essa pesquisa adotou termos de confidencialidade de dados (adultos e responsáveis), de consentimento livre e esclarecido (adultos e responsáveis), e de assentimento (crianças) em todas as avaliações com usuários. O questionário MEEGA+ [Group 2018] foi aplicado em sua versão original com os pais, professores e terapeutas para aferir o potencial educacional do jogo, e, com as crianças, foi utilizada uma versão simplificada elaborada pela autora com base no MEEGA+ original.

\section{Descrição e discussão dos resultados obtidos}

$\mathrm{Na}$ fase inicial do projeto foram utilizadas as recomendações de [AbleGamers 2018], [GAG 2018] e [Hussain et al. 2016].

Uma descrição geral dos jogos (escolher o que comer, escolher o que vestir, e escovar os dentes) e protótipos em papel foram apresentados para uma terapeuta ocu- 
VIII Congresso Brasileiro de Informática na Educação (CBIE 2019)

Anais dos Workshops do VIII Congresso Brasileiro de Informática na Educação (WCBIE 2019)

pacional da APAE - Alegrete/RS. Uma entrevista semiestruturada com questões abertas coletou feedback sobre a adequação da proposta.

A especialista considerou o jogo válido para ser utilizado com crianças com TEA e considerou que o jogo era fácil de aprender e utilizar. Adicionalmente, foi sugerido um novo tema de jogo para uma atividade considerada importante na vida diária: arrumar a mesa. Com este retorno, foi realizada a construção do protótipo refinado em papel dos jogos. Dois desses protótipos podem ser encontrados na Figura 2.

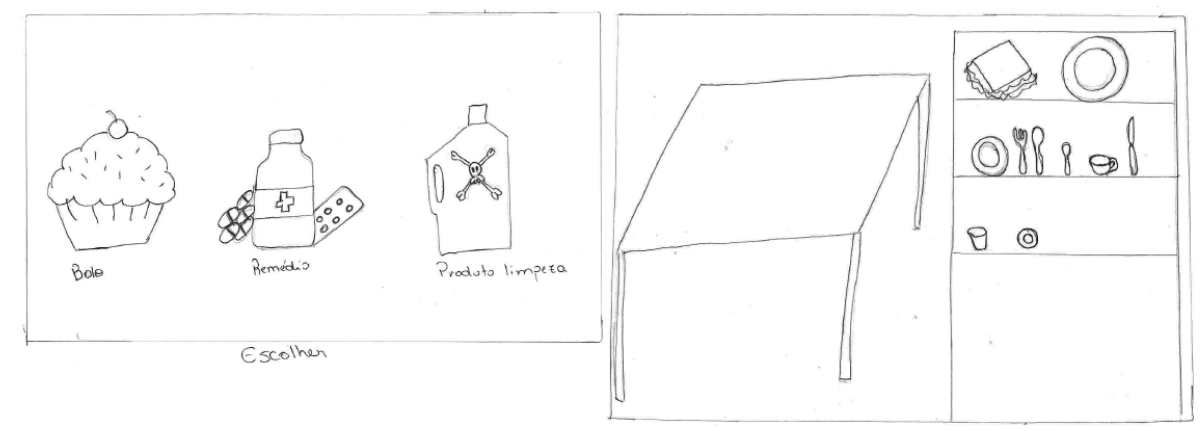

Figura 2. Protótipos dos jogos Hábitos de Alimentação e Segurança (esquerda) e Arrumar a Mesa (direita)

O desenvolvimento da primeira versão do jogo (com apenas um minijogo) teve seu tempo estimado em 208 horas, de acordo com as atividades pré-determinadas. Efetivamente, o desenvolvimento levou aproximadamente 189 horas. Esta primeira versão contou com a aplicação das diretrizes de acessibilidade encontradas durante a fase de pesquisa bibliográfica inicial e as recomendações da especialista consultada. Os sons e as imagens do jogo foram, em parte, criados pela autora dessa pesquisa e, em parte, obtidos de repositórios gratuitos da Internet.

Após o desenvolvimento do primeiro jogo, foram aplicados testes com usuários no consultório particular de uma fonoaudióloga que atende crianças com TEA. Estes testes foram realizados com três crianças participantes (todos do sexo masculino com idades entre 7 e 10 anos e com nível de autismo diagnosticado entre leve e moderado), utilizando um tablet de 9.6 polegadas com sistema operacional Android 4.4.

O protocolo de testes foi elaborado considerando especificamente as particularidades de comunicação e interação das crianças com TEA. A fonoaudióloga participou do teste como mediadora, pois possuía vínculo estabelecido com os participantes.

Durante a execução do primeiro teste foram detectados problemas com alta severidade que poderiam interferir na jogabilidade: (1) Tempo de exibição dos feedbacks muito longo: o tempo de exibição dos feedbacks estava inicialmente configurado para 5 segundos, fazendo as crianças ficarem impacientes. O tempo foi reduzido para 2 segundos. (2) Cliques inválidos durante a exibição dos feedbacks: as crianças continuavam pressionando objetos mesmo com a exibição dos feedbacks que cobriam os elementos na tela. Para resolver este problema, os objetos clicáveis que ficavam em segundo plano foram removidos durante a exibição dos feedbacks. Problemas menores cujos detalhes saem do escopo desse trabalho foram também detectados, registrados e corrigidos.

O desenvolvimento da segunda versão do jogo (contendo os dois minijogos que 
VIII Congresso Brasileiro de Informática na Educação (CBIE 2019)

Anais dos Workshops do VIII Congresso Brasileiro de Informática na Educação (WCBIE 2019)

compuseram a versão final) teve tempo estimado em 186 horas, sendo efetivamente concluído em 133 horas. Essa versão recebeu as modificações resultantes da análise dos problemas encontrados na avaliação inicial.

A segunda avaliação realizada contou com a participação de cinco crianças (uma participante do sexo feminino e quatro do sexo masculino, com idades entre 6 e 12 anos), sendo três testes realizados no consultório particular da fonoaudióloga e outros dois testes realizados na APAE - Alegrete/RS. A avaliação também contou com a participação de duas especialistas (uma fonoaudióloga e uma terapeuta ocupacional) e três adultos responsáveis por três crianças que participaram no consultório particular.

Este segundo teste avaliou as correções dos problemas anteriormente detectados, a inclusão do novo minijogo, a estrutura geral do aplicativo, e o potencial educativo do jogo. A versão final dos dois minijogos avaliados pode ser vista na Figura 3.

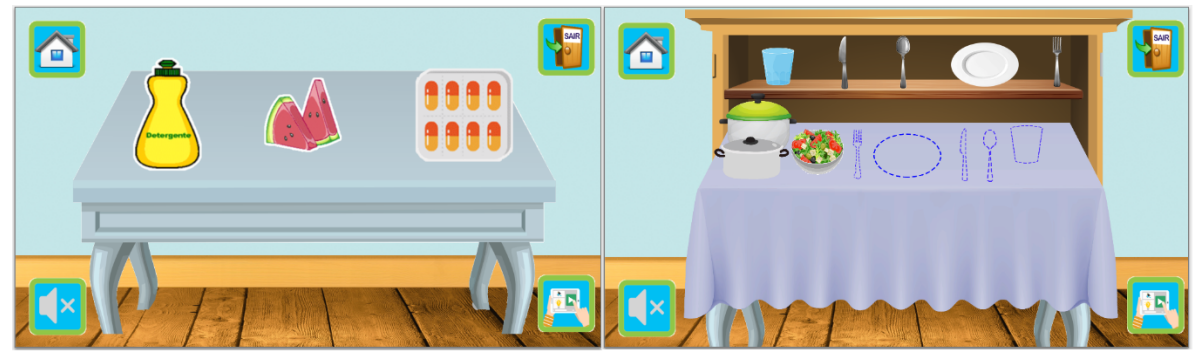

Figura 3. Versão final dos jogos Hábitos de Alimentação e Segurança (esquerda) e Arrumar a Mesa (direita)

$\mathrm{Na}$ avaliação final do jogo foram utilizados dois tablets, sendo um de 9.6 polegadas e outro de 7 polegadas. O maior foi utilizado com as crianças e o menor com os adultos. O teste ocorreu em dois ambientes separados, simultaneamente com a criança e seu responsável. A interação da criança com os jogos e a aplicação dos questionários com as crianças foram realizadas pela autora desse trabalho. Os adultos interagiram sozinhos com o jogo e responderam os questionários também sozinhos. Os testes com a fonoaudióloga e a terapeuta ocupacional foram acompanhados pela autora.

Para tabular os dados, foi utilizada a planilha de apoio do questionário MEEGA+ [Group 2018] com os pais e professores. Para os dados das crianças, foi utilizada uma planilha simplificada, elaborada com base no modelo original. Como resultado da tabulação, podemos destacar as dimensões de experiência do jogador das crianças, conforme a $\mathrm{Fi}^{-}$ gura 4, e a experiência do jogador dos adultos, conforme a Figura 5.

Em relação ao tópico sobre o que mais gostaram no jogo, as crianças disseram que gostaram mais "das figuras e imagens de comida". Não houve feedback significativo em relação ao que as crianças menos gostaram.

Além de responder às questões objetivas, os adultos destacaram como pontos fortes "a criatividade e a organização do jogo" e que "o jogo é capaz de ensinar brincando a comer e arrumar a mesa". Por fim, também sugeriram mudanças no jogo, como "mudança de fases", "usar outros temas além de alimentos" e "desenhos mais coloridos".

Durante o desenvolvimento do jogo foram adotadas diversas diretrizes de acessibilidade de [AbleGamers 2018, GAG 2018, Hussain et al. 2016] que incluíram: existência 
VIII Congresso Brasileiro de Informática na Educação (CBIE 2019)

Anais dos Workshops do VIII Congresso Brasileiro de Informática na Educação (WCBIE 2019)

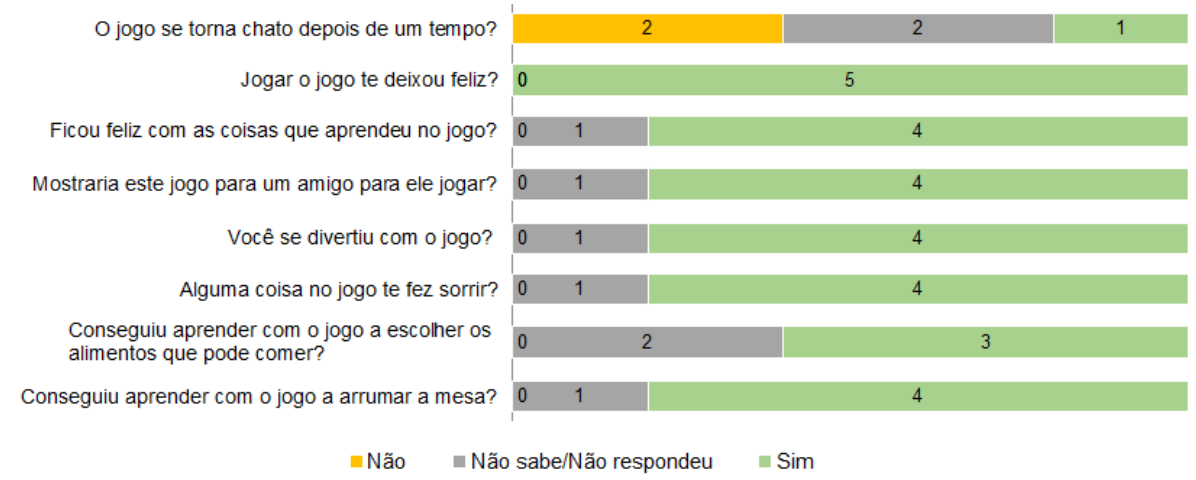

Figura 4. Fator de experiência do jogador das crianças

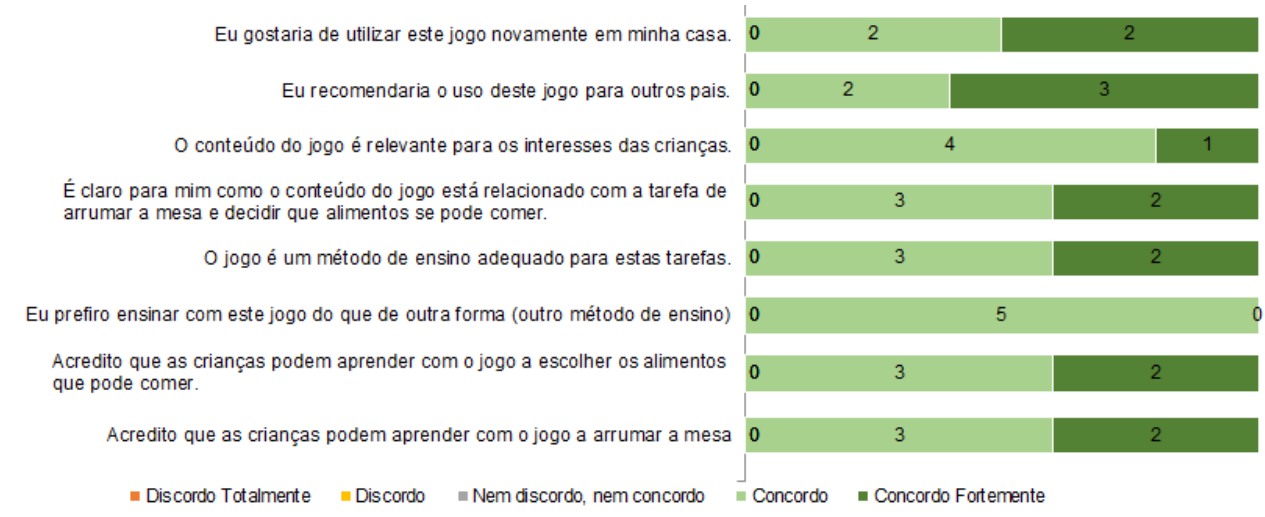

Figura 5. Fator de experiência do jogador dos adultos

de tutorial, vocabulário e fonte simples, vocabulário visual e textual compatíveis com mundo real, imagens e padrões não repetitivos, elementos interativos claros, evitar sons perturbadores, evitar eventos inesperados, informação em áudio, cores não são a única fonte de uma informação, ícones facilmente distinguíveis, ícones e botões com tamanho adequado, evitar distrações gráficas, navegação simples e sem tempo limite, feedback positivo e negativo multimodal e adequado, e prevenção de erros.

Após o desenvolvimento e a validação do jogo, foram obtidas novas recomendações a serem observadas ao desenvolver jogos para crianças com TEA: tempo de exibição de feedback precisa ser curto, tutoriais em vídeo não devem conter elementos que possam ser confundidos com a execução do próprio jogo, tela deve ter poucos botões, instruções diretas em frases curtas, e não usar sons ou músicas em volume alto.

\section{Conclusão}

Este trabalho possibilitou desenvolver um jogo mobile para aumentar a autonomia de crianças com Transtornos de Espectro Autista (TEA), retomando diretrizes e recomendações de acessibilidade para esse público e identificando novas.

A inclusão de especialistas no processo de desenvolvimento em entrevistas individuais permitiu ampliar a qualidade do produto final e sua eficácia junto ao público alvo. O uso de prototipação em papel também facilitou significativamente a comunicação técnica com especialistas. Os testes com crianças com TEA permitiram identificar peculiaridades de interação com esse público e exigiram reflexão sobre formas de contornar 
VIII Congresso Brasileiro de Informática na Educação (CBIE 2019)

Anais dos Workshops do VIII Congresso Brasileiro de Informática na Educação (WCBIE 2019)

barreiras por meio de comunicação alternativa ou mediadores. Ainda, foi possível aplicar um processo de software recentemente proposto - Personal Scrum [Costa 2016] - para desenvolvimento de jogos por desenvolvedores solitários.

O jogo desenvolvido está disponibilizado na Google Play Store ${ }^{6}$ para que possa ser utilizado com outras crianças além daquelas que foram envolvidas na pesquisa.

Limitações significativas enfrentadas durante o processo incluem (1) redução do escopo inicial do trabalho pela metade (foram desenvolvidos apenas dois jogos dos quatro jogos projetados) por conta de prazos para entrega do Trabalho de Conclusão de Curso, e (2) impedimento da realização de mais testes com estudantes na APAE - Alegrete/RS devido a uma enchente severa que afetou sua estrutura e impediu as aulas.

Com relação a trabalhos futuros, sugere-se (1) trabalhar com validações adicionais com especialistas em TEA, (2) realizar mais testes com crianças com TEA de diferentes níveis de severidade, (3) validar a qualidade psicométrica da versão adaptada do MEEGA+ para coletar dados de crianças com TEA, e, por fim, (4) desenvolver e validar os outros dois outros jogos previstos na proposta inicial e que não foram desenvolvidos.

\section{Referências}

AbleGamers, F. (2018). Includification. https : / /www . includification.com/.

Abrantes, K. (2010). A importância dos jogos didáticos no processo de ensino aprendizagem para deficientes intelectuais. http://www. fiepb.com.br.

Association, A. P. et al. (2014). DSM-5: Manual diagnóstico e estatístico de transtornos mentais. Porto Alegre, RS.

Barbosa, S. and Silva, B. (2010). Interação humano-computador. Elsevier Brasil, Rio de Janeiro, RJ.

Beck, R. G. (2017). Estimativa do número de casos de transtorno do espectro autista no sul do brasil. Master's thesis, Programa de Pós-Graduação em Ciências da Saúde, Universidade do Sul de Santa Catarina, Tubarão, SC.

Brandão, A. and Joselli, M. (2015). Jecripe 2: estimulação da memória, atenção e sensibilização fonológica em crianças com síndrome de down. In Proceedings of the XIV Brazilian Symposium on Games and Digital Entertainment, SBGAMES, volume 15, pages 518-525, Teresina, PI. IEEE.

Britto, T. C. P. (2016). Gaia: uma proposta de guia de recomendações de acessibilidade web com foco em aspectos do autismo. Master's thesis, Universidade Federal de São Carlos, São Carlos, SP. Acessado em 09/04/2018.

Cardoso, M. (2014). Deficiência mental: conhecer para incluir. In Santarosa, L. M. C., Conforto, D., and Vieira, M. C., editors, Tecnologia e Acessibilidade: Passos em direção à inclusão escolar e sociodigital, pages 35-42. Evangraf, Porto Alegre, RS.

Costa, K. R. N. (2016). Personal scrum: uma alternativa ágil para desenvolvimento de indie games. Trabalho de conclusão de curso, Bacharelado em Engenharia de Software, Universidade Federal do Pampa, Alegrete, RS.

\footnotetext{
${ }^{6}$ Disponível em http://bit.ly/tea juda-googleplay e compatível com dispositivos Android (smartphone e tablets) de versão 4.4 ou superior.
} 
VIII Congresso Brasileiro de Informática na Educação (CBIE 2019)

Anais dos Workshops do VIII Congresso Brasileiro de Informática na Educação (WCBIE 2019)

Durango, I., Carrascosa, A., Gallud, J. A., and Penichet, V. M. R. (2015). Using serious games to improve therapeutic goals in children with special needs. In Proceedings of the 17th International Conference on Human-Computer Interaction with Mobile Devices and Services Adjunct, MobileHCI '15, pages 743-749, New York, NY. ACM.

Faders and do Rio Grande do Sul, A. L. (2011). Dicas de convivência com pessoas com deficiência. http://www.portaldeacessibilidade.rs.gov. br/uploads/1309442549Cartilha_Dicas_de_Convivencia_PCD_ Faders_e_AL_RS.pdf.

GAG (2018). Game accessibility guidelines. http:// gameaccessibilityguidelines.com/.

Group, S. Q. (2018). Meega+, systematic model to evaluate educational game. http://www.gqs.ufsc.br/ meega-a-model-for-evaluating-educational-games/.

Hussain, A., Abdullah, A., Husni, H., and Mkpojiogu, E. O. (2016). Interaction design principles for edutainment systems: Enhancing the communication skills of children with autism spectrum disorders. Revista Tecnica de la Facultad de Ingenieria Universidad del Zulia, 39(8):45-50.

IBGE (2010). Resultados preliminares da amostra - censo ibge. http://www . pessoacomdeficiencia.gov.br/app/indicadores/censo-2010.

Kolakowska, A., Landowska, A., and Karpienko, K. (2017). Gyroscope-based game revealing progress of children with autism. In Proceedings of the 2017 International Conference on Machine Learning and Soft Computing, ICMLSC '17, pages 19-24, New York, NY, USA. ACM.

Moreira, K. d. S., Silva, M. S. A., and Melo, C. d. M. A. C. (2016). Escola x autismo percepção dos pais. https://editorarealize.com.br/revistas/ fiped/resumo.php?idtrabalho=863.

Organization, W. H. (2017). International classification of functioning, disability and health - icf browser. http://apps.who.int/classifications/ icfbrowser/.

Rabin, S. (2011). Introdução ao Desenvolvimento de Games, volume 1. Cengage Learning, São Paulo, SP.

Ribeiro, P. C., de Araujo, B. B. P. L., and Raposo, A. (2014). Comfim: a cooperative serious game to encourage the development of communicative skills between children with autism. In Computer Games and Digital Entertainment (SBGAMES), 2014 Brazilian Symposium on, pages 148-157, Porto Alegre, RS. IEEE.

Salen, K. and Zimmerman, E. (2012). Regras do jogo: fundamentos do design de jogos, volume 1. Blucher, São Paulo, SP.

Sampieri, R. H., Collado, C. F., and del Pilar Baptista Lucio, M. (2013). Metodologia de Pesquisa. Penso, Porto Alegre, RS, 5 edition.

Sommerville, I. (2011). Engenharia de Software. Pearson, São Paulo, SP. 